\title{
Open-heart palliative surgery for pulmonary atresia with ventricular septal defect and hypoplastic pulmonary arteries
}

\author{
GIANCARLO CRUPI, GIUSEPPE LOCATELLI, MASSIMO VILLANI, \\ ROBERTO TIRABOSCHI, AND LUCIO PARENZAN
}

From the Department of Cardiac Surgery, Ospedali Riuniti, Bergamo, Italy

Crupi, G, Locatelli, G, Villani, M, Tiraboschi, R, and Parenzan, L (1978). Thorax, 33, 625-628. Open-heart palliative surgery for pulmonary atresia with ventricular septal defect and hypoplastic pulmonary arteries. The surgical management of pulmonary atresia with ventricular septal defect (VSD) and hypoplastic pulmonary arteries poses difficult problems. Adequate palliation was achieved in two such patients by restoring continuity between the right ventricle and the pulmonary arteries by means of a woven Dacron patch.

The VSD was left unrepaired. Angiography performed one month after operation showed the reconstructed pulmonary outflow tract to be widely patent and the pulmonary arteries to be enlarged. This approach may allow subsequent total correction by closure of the VSD in these hopeless patients.

Total correction in patients with pulmonary atresia and ventricular septal defect (VSD) is now feasible with a high probability of success (Olin et al, 1976). However, only a few reach the ideal age for corrective surgery without a palliative operation. In most patients severe cyanosis is present, and death may occur at an early age. The accepted management for these infants has been the creation of a systemic-to-pulmonary artery anastomosis. A different type of palliation previously used by Gerbode (Pacifico et al, 1977) has recently been considered for those patients with pulmonary atresia and VSD and hypoplastic pulmonary arteries (Gill et al, 1977). We describe two such patients in which right ventricular-pulmonary artery continuity was restored by placement of a Dacron woven patch with the aid of deep hypothermia, leaving the VSD open.

\section{Case reports}

CASE 1 A 4-month-old boy was admitted to our department in August 1976. He had been cyanotic since birth but had no cyanotic spells. Catheterisation at that time showed pulmonary atresia with VSD and hypoplastic right and left pulmonary arteries (fig 1(a)). The only source of pulmonary blood supply was from two systemic-
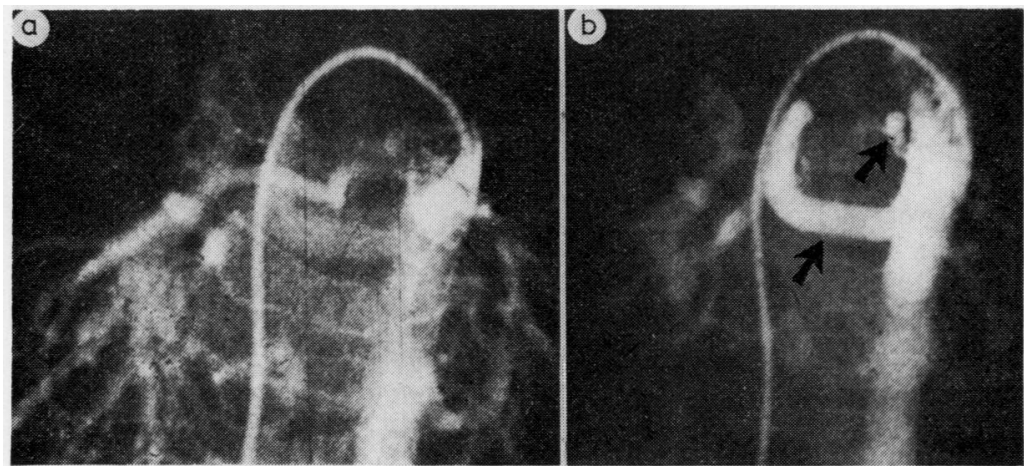

Fig 1 Preoperative anteroposterior angiogram showing (a) hypoplastic confluent pulmonary arteries opacified through (b) two systemic-pulmonary collateral arteries (arrowed). Injection was made into upper descending aorta. 

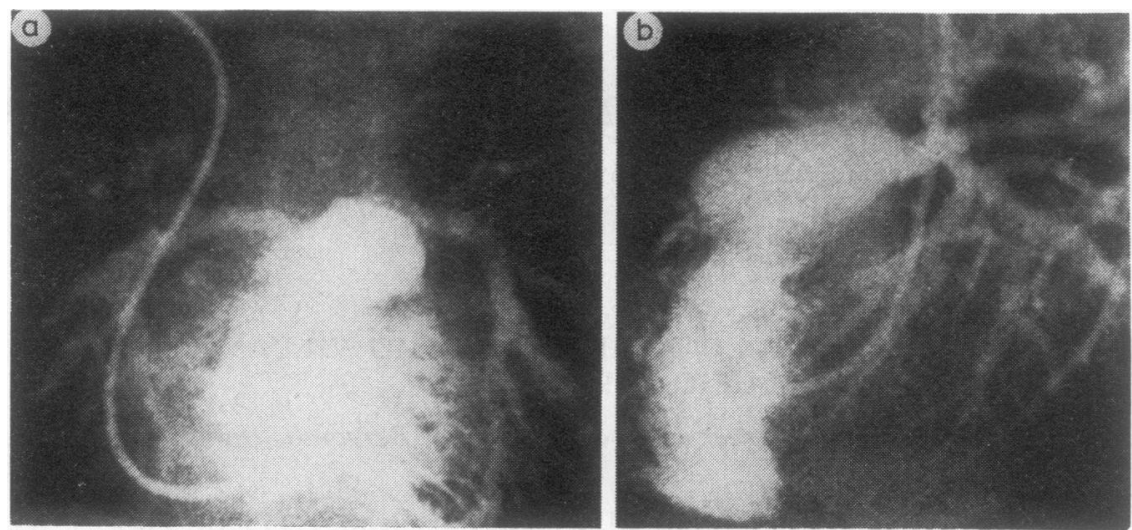

Fig 2 Postoperative anteroposterior (a) and lateral (b) right ventriculogram shows reconstructed pulmonary outflow tract. Pulmonary arteries are slightly enlarged.

(b) is a later frame.

pulmonary collateral arteries arising from the upper descending aorta (fig 1(b)). Surgical treatment was deferred because symptoms were not severe and the peripheral arterial oxygen saturation was $80 \%$.

The child was readmitted one year later because of progressive cyanosis and failure to thrive. Operation was performed through a median sternotomy using deep hypothermia and cardioplegia with Bretschneider solution, which provided adequate operating conditions. There was pulmonary valve atresia. The pulmonary trunk measured $5 \mathrm{~mm}$ and the pulmonary arteries $3 \mathrm{~mm}$ in diameter. A longitudinal incision made over the infundibulum of the right ventricle was carried across the pulmonary annulus and then extended to the bifurcation of the pulmonary trunk. Muscular tissue interposed between the blind-ending right ventricle and the pulmonary trunk was excised, and a patch made from a $14 \mathrm{~mm}$ woven Dacron tube was sutured to the edges of the incision. The postoperative course was uneventful, and the peripheral arterial oxygen saturation increased from $68 \%$ to $87 \%$. Angiography performed one month later showed the right ventricular outflow tract to be patent and the pulmonary arteries to be slightly enlarged (fig 2(a) and (b)).

CASE 2 The second patient was a 3-year-old boy. A cardiac murmur was present at birth, and cyanosis was noticed at the age of 5 months. Since that time he had experienced progressive cyanosis and lethargy. Cardiac catheterisation in January 1976 showed pulmonary atresia with VSD and hypoplastic pulmonary arteries. A large systemicpulmonary collateral artery arising from the upper right-sided descending aorta was the only source $\stackrel{\Phi}{-}$ of pulmonary blood supply to both the lungs $\vec{\varphi}$ (fig 3). Surgical treatment at that time was not advised. In September 1977 the patient was readmitted and underwent operation through a median sternotomy. Deep hypothermia and cold cardioplegia were used. There was a gap of continuity of about $1 \mathrm{~cm}$ between the patent pulmonary $\frac{\partial}{\mathbb{D}}$ trunk and the right ventricular outflow tract while $\triangle$ the right and left pulmonary arteries measured $\overrightarrow{\overrightarrow{0}}$ about $4 \mathrm{~mm}$ in diameter. A longitudinal incision 3 was made over the infundibulum of the right ventricle, and the pulmonary trunk was divided up? to the bifurcation. A small muscular resection was carried out along the border of the ventriculotomy,

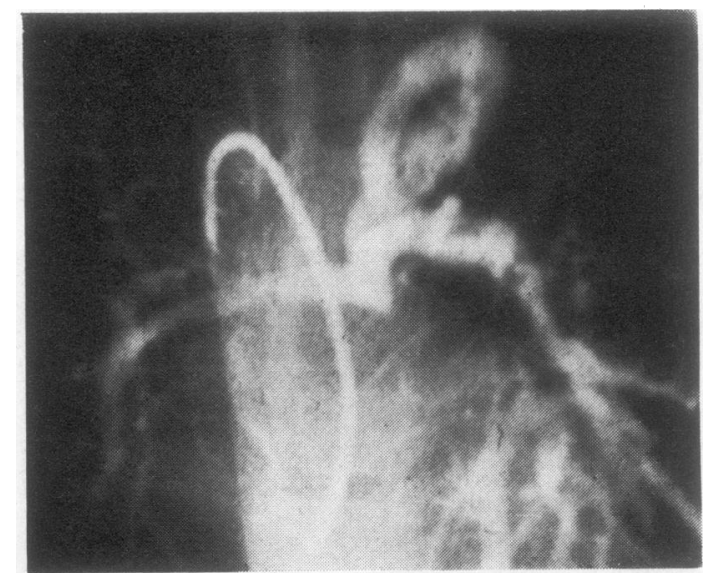

Fig 3 Preoperative anteroposterior angiogram showing hypoplastic confluent pulmonary arteries opacified through a large systemic-pulmonary collateral arising from a right-sided thoracic aorta. 
and a long woven Dacron patch made from a $16 \mathrm{~mm}$ tube was sutured to the entire length of the combined cardiotomy-arteriotomy. The patient required assisted ventilation for 72 hours, but otherwise the postoperative course was satisfactory. The peripheral arterial oxygen saturation increased from $65 \%$ to $85 \%$. Angiography one month later showed the pulmonary outflow tract to be widely patent and the pulmonary arteries to be clearly enlarged (fig 4 ).

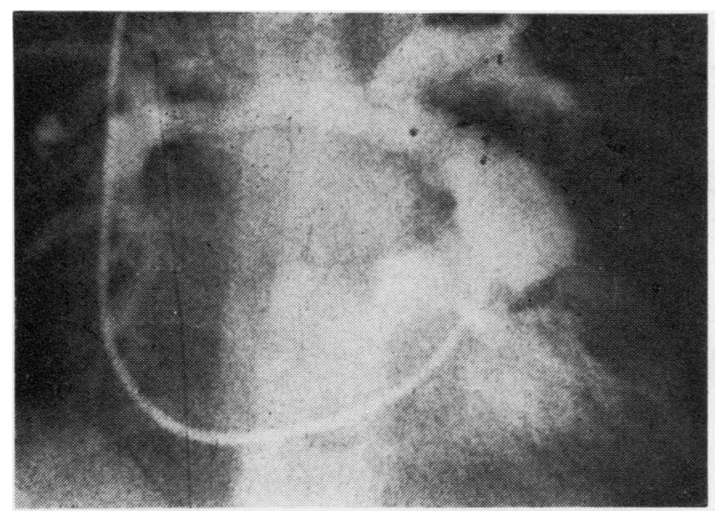

Fig 4 Postoperative anteroposterior angiogram showing significant enlargement of pulmonary arteries. Pulmonary outflow tract is widely patent.

\section{Discussion}

Successful total repair of pulmonary atresia with VSD in patients below 6 months of age has been reported by Barratt-Boyes (1973) and Ebert et al (1976). The age of the patients and the size of the pulmonary arteries should always be carefully evaluated in planning total correction for this anomaly (McGoon et al, 1975). If possible intracardiac repair should be delayed until the age of 3 to 4 when an adult-size conduit can be inserted if necessary (Olin et al, 1976; Norwood et al, 1977)

If the pulmonary arteries are too hypoplastic to permit corrective operation the creation of a systemic-to-pulmonary artery anastomosis is indicated to relieve cyanosis and promote development of the proximal pulmonary vasculature. In a few patients, however, the pulmonary arteries are so small that a systemic-to-pulmonary artery shunt may not be successful, either because the flow through the anastomosis would be too small or because of distortion or kinking of the minute right or left pulmonary artery. In these circumstances, creation of right ventricular-pulmonary artery continuity while leaving the VSD open can provide more physiological palliation.

This approach was apparently first used by Gerbode (1977) and has recently been used with success by Gill et al (1977). Norwood et al (1976) used a similar approach but closed the VSD with a perforated patch.

Our experience consists of two patients with pulmonary atresia and VSD in whom the pulmonary arteries measured less than $5 \mathrm{~mm}$ in diameter.

Continuity between the right ventricle and the patent pulmonary arteries was restored by placing a woven Dacron patch across the atretic pulmonary valve while the VSD was left open (fig 5). The patch was tailored from a Dacron woven tube whose diameter was chosen according to the data on the minimal acceptable valve ring diameter reported by Pacifico et al (1977). Systemicpulmonary collateral arteries, which in both cases represented the only source of pulmonary blood supply to both lungs, were not ligated. Deep hypothermia and cold cardioplegia by infusing Bretschneider solution at $4^{\circ} \mathrm{C}$ into the aortic root provided adequate operating conditions. The blood flow was temporarily reduced to $0.51 / \mathrm{min} / \mathrm{m}^{2}$ for up to 20 minutes if the intracardiac return of blood became excessive.

The postoperative course was satisfactory, and in both patients the peripheral arterial oxygen saturation increased, indicating an increased pulmonary blood flow through the newly reconstructed pulmonary outflow tract. Cardiac catheterisation one month later showed the pulmonary outflow tract to be widely patent in both cases and the proximal pulmonary arteries to be clearly enlarged in case 2. Three months later these two patients are in good general condition and only slightly cyanotic on effort. The clinical course of these patients will determine the optimal timing for further catheterisation. At that time corrective surgery by closure of the VSD will be decided according to the size of the pulmonary arteries.

Our experience endorses the opinion of Gill et al (1977) that the creation of continuity between right ventricle and pulmonary artery in patients with pulmonary atresia, VSD, and hypoplastic pulmonary arteries offers several advantages compared with a systemic-to-pulmonary artery anastomosis. Kinking of the pulmonary arteries is avoided as they are not dissected and mobilised, and the pulmonary blood flow is distributed equally between both pulmonary arteries providing optimal conditions for their subsequent enlargement.

Though the long-term results of this approach 

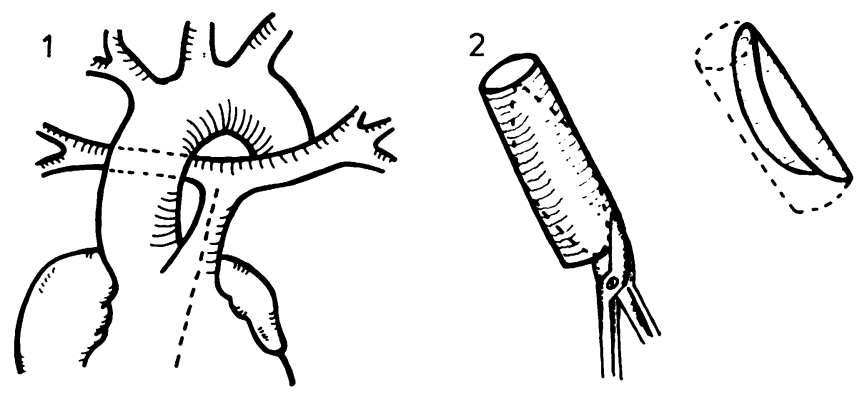

Fig 5 Diagrammatic illustration of type of surgical repair used. 1 Incision from right ventricle to bifurcation of pulmonary trunk
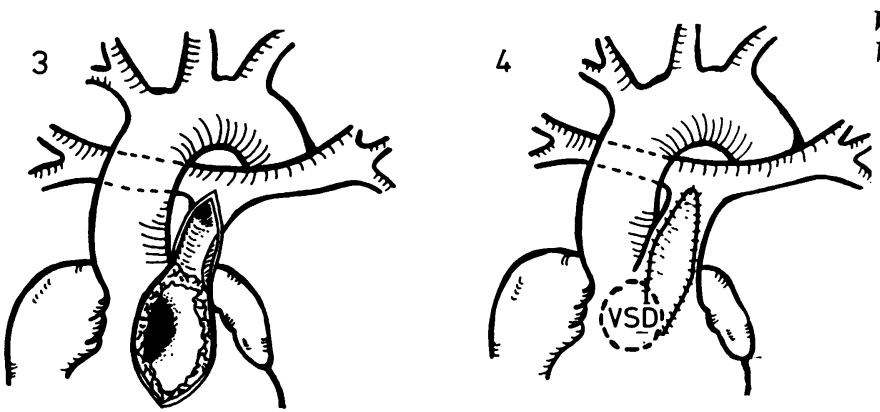
through atretic area. 2 Tailoring of patch. 3 $V$ iew of prepared outflow tract showing VSD. 4 Complete patch graft.

are as yet undetermined we hope these patients may undergo later correction by closure of the VSD.

\section{References}

Barratt-Boyes, B G (1973). Primary definitive intracardiac operation in infants: Tetralogy of Fallot. In Advances in Cardiovascular Surgery, edited by J W Kirklin, pp 163-166. Grune and Stratton, New York.

Ebert, P A, Robinson, S J, Stanger, P, and Engle M A (1976). Pulmonary artery conduits in infants younger than 6 months of age. Journal of Thoracic and Cardiovascular Surgery, 72, 351-356.

Gerbode, F, in discussion of Pacifico et al (1977).

Gill, C G, Moodie, D S, and McGoon, D C (1977). Staged surgical management of pulmonary atresia with diminutive pulmonary arteries. Journal of Thoracic and Cardiovascular Surgery, 73, 436-442.

McGoon, D C, Baird, D K, and Davies, G D (1975). Surgical management of large bronchial collateral arteries with pulmonary stenosis or atresia. Circulation, 52, 109-118.
Norwood, W I, Rosenthal, A, and Castaneda, A R (1976). Tetralogy of Fallot with acquired pulmonary atresia and hypoplasia of pulmonary arteries. Report of surgical management in infancy. Journal of Thoracic and Cardiovascular Surgery, 72, 454-457.

Norwood, W I, Freed, M D, Rocchini, A P, Bernhard, W F, and Castaneda, A R (1977). Experience with valved conduits for repair of congenital cardiac lesions. Annals of Thoracic Surgery, 24, 223-232.

Olin, C G, Ritter, D C, McGoon, D C, Wallace, R B, and Danielson, G K (1976). Pulmonary atresia: surgical considerations and results in 103 patients undergoing definitive repair. Circulation, suppl III, 54, 35-40.

Pacifico, A D, Kirklin, J W, and Blackstone, E H (1977). Surgical management of pulmonary stenosis in tetralogy of Fallot. Journal of Thoracic and Cardiovascular Surgery, 74, 382-395.

Requests for reprints to: Dr G Crupi, Department of Cardiac Surgery, Brompton Hospital, Fulham Road, London SW3. 